\title{
Staffs awareness of abuse in health care varies according to context and possibilities to act
}

\author{
Katarina Swahnberg and Barbro Wijma
}

\section{Linköping University Post Print}

N.B.: When citing this work, cite the original article.

Original Publication:

Katarina Swahnberg and Barbro Wijma, Staffs awareness of abuse in health care varies according to context and possibilities to act, 2011, JOURNAL OF PSYCHOSOMATIC OBSTETRICS AND GYNECOLOGY, (32), 2, 65-71.

http://dx.doi.org/10.3109/0167482X.2011.555021

Copyright: Informa Healthcare

http://informahealthcare.com/

Postprint available at: Linköping University Electronic Press

http://urn.kb.se/resolve?urn=urn:nbn:se:liu:diva-68904 
TITLE: Staff's awareness of abuse in health care varies according to situation and possibilities to act.

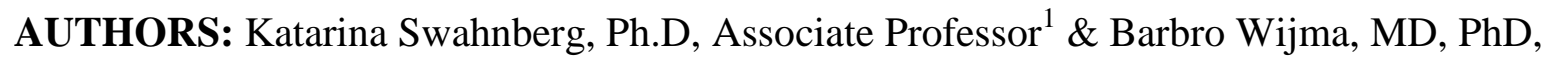
Professor. $^{1,2}$

1.Division of Gender and Medicine, Department of Clinical and Experimental Medicine, Faculty of Health Sciences, Linköping University, 58183 Sweden.

2. Dept. of Obstetrics and Gynaecology, County Council of Östergötland, Linköping, 58183 Sweden.

SHORT TITLE: Abuse in health care

KEYWORDS: nurses; midwives; nursing; ethics; patient safety; quality of care; qualitative method.

ACKNOWLEDGEMENTS

This work was supported by The Swedish Research Council (K2007-70X-20536-01-3) and the Health Research Council in the Southeast of Sweden (FORSS-8987).

The authors report no conflicts of interest.

CORRESPONDING AUTHOR: Katarina Swahnberg PhD, Division of Gender and

Medicine, Department of Clinical and Experimental Medicine, Faculty of Health Sciences, Linköping University, S-581 83 Linköping, Sweden.

Email: katarina.swahnberg@liu.se

Phone; + 460101033191 


\section{ABSTRACT}

Objectives: The aim of this study was to explore awareness of abuse in health care (AHC) from a staff perspective.

Patient evaluation studies often focus patient satisfaction, and serious negative experiences might therefore be obscured. In our research we have found that AHC is commonly reported by male and female patients, when asked for in a strait way, but so far no intervention studies against AHC have been published. Investigating staff's awareness of AHC is our first step toward developing interventions against AHC.

Study design: Data was collected at a Swedish clinic of obstetrics and gynaecology. Qualitative interviews with 21 informants were analysed with constant comparative analyses. Results: The core category - 'Staff's awareness of AHC varies according to context and possibilities to act' - was derived from the interaction between five categories; Moral imagination, Relativism, Explanations, Dissociation from AHC and Acting against AHC. Awareness of AHC was not a permanent state that did/did not exist as all participants displayed both high and low awareness; depending on the situation.

Conclusion: Staffs awareness depends on more than personal characteristics; therefore AHC interventions have to target individual behavior as well as cultures and structures in health care.

KEYWORDS: ethics; patient safety; quality of care; qualitative method; abuse in health care; education. 


\section{INTRODUCTION}

Why is it important to know how patients perceive the health care they receive? There are several ethical reasons, but also humanitarian and practical ones; namely to behold the patients' confidence, to avoid treatment-seeking delays, to increase patients' compliance and avoid suffering; and of course the overall responsibility to improve health care.

For these reasons, various kinds of patient evaluation studies have been conducted since the 1960s. The research field has not only offered methodological challenges, it has also been discussed from a content perspective: what should be evaluated?

Patient evaluation studies have often been operationalized as patient satisfaction studies (1).

The theoretical ground of the concept patient satisfaction has been questioned because of poor conceptual and theoretical development (1-5). Some of these concerns have emerged from the positive evaluations that satisfaction studies regularly produce $(2,6)$, thereby concealing dissatisfaction as well as traumatic experiences. There has also been a massive critique against quantitative questionnaires' ability to measure personal experiences of care $(1-3,7$ 10). The journey has gone from evaluating patient satisfaction $(1,3,11,12)$ to various forms of patient dissatisfaction $(6,13,14)$ and, lately, abuse in health care $(\mathrm{AHC})(15)$.

AHC could be defined as any act perceived as abusive by the patient in any health care setting. AHC has been operationalized by concrete examples in The NorVold Abuse Questionnaire (NorAQ) (Table 1). Estimated prevalence among Nordic gynecology patients answering NorAQ ranged between 13 and 28 percent (15).

In interview studies patients recognized $\mathrm{AHC}$ as a frequent and serious problem $(8,16-18)$, while staff generally had difficulties to remember any concrete episodes of AHC, even though they believed that AHC occurred frequently even at their clinic (19). Our research question was based on this paradox; to what extent is staff aware of AHC? 
Table 1. Questions in NorAQ about abuse in health care.

\begin{tabular}{|l|l|}
\hline $\begin{array}{l}\text { Mild } \\
\text { abuse }\end{array}$ & $\begin{array}{l}\text { ABUSE IN HEALTH CARE } \\
\text { felt that someone exercised blackmail against you or did not show respect for } \\
\text { your opinion - in such a way that you were later disturbed by or suffered from the } \\
\text { experience? }\end{array}$ \\
$\begin{array}{l}\text { Moderate } \\
\text { abuse }\end{array}$ & $\begin{array}{l}\text { Have you ever experienced that a "normal" event, while visiting health services } \\
\text { suddenly became a really terrible and insulting experience, without you fully } \\
\text { knowing how this could happen? }\end{array}$ \\
Severe abuse & $\begin{array}{l}\text { Have you experienced anybody in health service purposely - as you understood - } \\
\text { hurting you physically or mentally, grossly violating you or using your body and } \\
\text { your subordinated position to your disadvantage for his/her own purpose? }\end{array}$ \\
\hline & $\begin{array}{l}\text { ANSWER ALTERNATIVES (THE SAME FOR ALL QUESTIONS) } \\
\text { as a child and as an adult }\end{array}$ \\
\hline
\end{tabular}

Note: The section about AHC was introduced as follows: The following questions deal with abuse in health services. We ask you to mark if you have experienced any of the following events; as a child or as an adult. If you answer yes to any of the questions 41-43 we call it - in this study - that you have been subjected to abuse in health services. 


\section{METHOD}

\section{Materials and procedures}

Eligible for the present study were 140 staff members at a Swedish clinic of obstetrics and gynecology. Informants were recruited to represent all staff categories, different age groups and male/female staff, but else at convenience. Twenty-four staff members were asked and three declined participation. There was a majority of female employees at the clinic, mirrored in our sample of 16 females and five males (all gynecologists): three administrators/secretaries, six midwifes, seven gynecologists, and five auxiliaries. In spring 2007 altogether 21 interviews were conducted by the two authors in silent rooms at the hospital. The semi structured interviews lasted in average 60 minutes, and was based on a general interview guide prepared by the two authors (Table 2) (20-22). Interviews were taperecorded and transcribed verbatim by a secretary.

\section{Table 2. Interview guide}

\section{THEMES FOR THE INTERVIEW}

How do you like your workplace?

Are patients being abused in heath care?

When I say abuse in health care, what is the first thing that comes to your mind?

Who is the agent when abuse in health care occurs?

Who is being abused in health care?

How does abuse in health care occur?

How can health care staff intervene against abuse in health care? 
Concerning translation of the concept AHC, the Swedish word 'Övergrepp' and 'kränkning' has been used synonymously and been translated to 'abuse' in English.

Written informed consent was obtained from all participants before the interviews started. Our request to conduct the study had been approved by the local ethical committee (Registration number 194-06).

\section{Data analysis}

Constant comparative analysis was used to process data (23-25). The transcribed interviews were analyzed line by line according to Glaser's scheme of open coding to generate substantive codes, i.e. words or sentences with a relationship to the research question $(23,26)$. The substantive codes were constantly compared between interviews to generate new substantive codes and categories. The relationship between the categories was analyzed and a core category that answered our research question was identified (26). Results from the analysis are summarized in Figure 1. To facilitate the comprehension for readers, the wording of substantive codes is not literal in Figure 1. No new categories emerged after eight interviews. 
Figure 1. Core category, categories and substantive codes answering the research question; to what extent is staff aware of abuse in health care?

Staff's awareness of abuse in health care varies according to context and possibilities to act

High awareness

Acting against $\mathrm{AHC} 17$

Dares to act 9

A too sensitive issue 10
Moral imagination 20

Examples of AHC from a patient perspective 14

Show consideration for patients 5

Show respect for patients 10

Understand patient's weak position 7

\section{Relativism 17}

Abuse against staff 13

High expectations among patients 10

Explanations 20

Subjective experiences among patients 15

Staff lacks time 8

Organisational faults 8

Staff had a bad day 5

No medical error 4

Dissociation from AHC 21

Regards AHC unavoidable 6

Regards AHC unintentional 16

Takes self-righteous stance 6

Does not remember examples 11

Questions \& analyses the word AHC 6

\section{Low awareness}

Note: The number of interviews represented in each substantive code/category is displayed after each substantive code/category (in total 21 interviews). 


\section{RESULTS}

The analysis shows that an individual's awareness of AHC is best illustrated as a continuum in constant movement, rather than being something a person has or has not. All informants gave examples and were reasoning on a scale from low to high awareness of AHC during the interviews. This continuum had four "platforms", representing decreasing degrees of awareness: 1) Moral imagination, 2) Relativism, 3) Explanations, and 4) Dissociation from AHC. Moral imagination represented the highest degree of awareness of AHC found in this study. Altogether there were five categories; the fifth category represented Acting against AHC (Figure 1).

\section{Moral imagination}

The category Moral imagination was based on staff members' ability to use a patient perspective and to be empathic. Examples in this category can be divided into own experiences, examples when other staff was considered to be abusive toward a patient, or most commonly, merely general examples. Some actions were without doubt considered to be wrong by the informants, e.g. that staff members often were reading patient records and talking about patients, even if they were not involved in the case, or that they were talking about other patients or their own private life, while helping a patient.

The examples given were often described by the informants as 'small things' that were important to be aware of to avoid AHC, e.g. how to look at, talk to or touch a patient. Moral imagination was furthermore based on awareness of the power asymmetry between patients and staff; i.e. the subordinated patient position and the superior staff position: 'They 
come because they need help...the patient is in reality subordinated...the hospital and staff really, and we have more power in a way.'(Auxiliary nurse)

Especially female staff often referred to the exposed and uncovered position held by a patient during the pelvic examination. They emphasized how important it was to cover the patient, and they knew exactly how they would feel in the same situation.

'...Sometimes I feel when I have to run out...to fetch something and she lies there and he sits down there...the gynecologist... and talks between her legs. You know! I think that's horrible! (sigh) Then I have to look for the midwife who will unlock a cupboard to get the medicine out...(catches a deep breath). I don't know how fast I want to get that medicine out of there you know because I know that she shouldn't lie like that (in a high voice). I think that's a little...I feel that in this situation she must feel abused.'(Auxiliary nurse)

\section{Relativism}

On a lower level of awareness of AHC, things became more relative and it was obvious that staff members were also concerned about their own feelings and experiences of being abused by patients, as well as by other staff members. They acknowledged that patients experienced AHC, but they also scrutinized their own vulnerability alongside the patients' as if they were relative to each other. It was mentioned that staff members at a gynecology clinic were more at risk for strong negative reactions from patients, because they were dealing with more sensitive issues than other specialties. Furthermore, negative reactions from patients were sometimes regarded not only unpleasant but also unfair:

'...staff members can also be abused so to say. The patient might say something too...question ...someone's competence...That person might feel really abused. There are 
...well...unpleasant patients so to say...and you try to do your best, you know. Everybody is doing their...best, you know...well, and still you are just told off... and then ...then you get sad and you feel abused somehow. (Auxiliary nurse)

Staff being abused by a patient was understood from an ill patient's perspective, e.g. a patient looking for a scapegoat when being in a state of crisis. But the understanding of patients' abusive behavior was often easier to handle on an intellectual than on an emotional level. The informants understood the patient's reaction but they were still frustrated because they knew they had done everything right, and therefore felt offended or unfairly treated when patients were abusive towards them.

Furthermore, many informants thought that the patient position had been strengthened and that some patients took advantage of that, and were more demanding nowadays; which had made it more difficult to work in health care. Higher expectations on the health care in the society where also proposed to make patients believe that they could demand more. 'When ...(they)...finally get their appointments, they expect miracles...' one gynecologist commented.

Another informant meant that health care had participated in creating these expectations, i.e. that health care can solve every problem, and suggested that this lack of clarity facilitates for AHC to occur. If patients knew what to expect from health care, they would not so easily feel abused, this informant concluded.

\section{Explanations}

There were many general explanations, not necessarily based in own experiences, of how abuse arise in health care, e.g. that AHC is connected to personality in patients as well as staff. The majority thought of AHC as a subjective experience, i.e. something that is abusive 
to one person might not be abusive to another person. More than two thirds of the informants argued that some people feel abused more easily than others, and that a patient who experiences AHC might choose to interpret things in a negative way.

Pregnant women were used as one example of patients being very sensitive and prone to negative interpretations.

Even when AHC was considered a subjective experience, some explanations were based on an additional notion of 'vulnerable patients'. For instance, patients who experience AHC might have had a bad experience in health care earlier or experienced other kinds of abuse earlier in life, making them more vulnerable in new health care encounters. Some thought that patients who belong to certain groups, such as e.g. immigrants or drug addicted were more exposed to AHC. The former because of ignorance among staff when it came to their customs, but also because of language and other cultural barriers, and the latter because they were often looked down on as human beings.

Stress and time constraint was one explanation and organizational structures and routines were also believed to create AHC. Different kinds of staff categories claimed that being interrupted in their work by colleagues made them irritated in a way that could influence their work with patients negatively. It could also be a matter of how well patients and staff got along, or depending on if the health care provider had a good or a bad day.

\section{Dissociation from AHC}

Staff appreciated the openness and the warm atmosphere at the clinic. They felt 'like a family' and thought that they were at the front edge when it came to behaving well toward patients. At the same time they said that they knew that $\mathrm{AHC}$ was common; even at their workplace. Some thought that it was inevitable that some patients would experience AHC. Some took it even further and said that AHC will always exist. 
When asked, more than every second informant found it difficult to come up with concrete examples of AHC (from their own experience or from what they had heard of).

Some informants were very critical toward the term 'abuse', and meant that there was a risk that it was used too often. There seemed to be a worry that the word abuse had undergone inflation, and that it was not used in a proper way anymore.

The majority thought that staff members were not aware when AHC happened, and that there was no intention to harm the patient. And still, they believed that AHC happened daily without the staff recognizing it, and that it was a matter of routines, i.e. getting too used to procedures and forgetting that they are new to most patients. This notion was very well described by one informant who gave an example of how this could happen. This example involves two levels of awareness; first the informant is not aware at all, but after having been made aware by the patient, the informant finds a good solution. Thus this example could illustrate both the Dissociation from AHC and Moral imagination categories.

'...I experienced it as a routine thing just to check that there were no problems...it was inbetween two other patients and a lot to do at the delivery ward. Well, then I think the door...I think it did not really close so there was a narrow opening I didn't notice. And then she was examined and there was some pain when I examined her (silence). Everything looked fine and so I told her it looks fine and everything is all right...and then I thought that, you know it was done. But afterwards the midwife told me that she had reacted and that this patient had felt abused in some way...So I went back to talk to her and I sat with her for a long time... so she was...very satisfied after that, but the thing was that she had thought of this...for her this (examination) was a big thing...something that I thought of as a routine thing I did inbetween two other patients... and I think that's the way it is for some people when they say 
that they have been abused. It's simply that you have two different images of what's happening.'(Gynecologist)

\section{Acting against AHC}

This category presumes some awareness of AHC. However, it became obvious that awareness, even if it was a necessary condition, was not enough to induce acting against AHC.

For example, it was considered important but difficult to tell a colleague that (s)he e.g. did something wrong to a patient. Acting against AHC was describes as a sensitive issue that demanded courage. 'I have not learned that yet' an experienced midwife commented. Maybe acting has to be learnt, but it might also be a question of personality, some informants had no option but to act, while others were more hesitant, or hierarchy; suggestions that were put forward in other interviews.

It was emphasized that openness between staff could facilitate behavioral change, and if the person was not made aware of being abusive to patients, (s)he would never learn. Someone even said that they wanted to know about their mistakes so that they could improve. However, this wish for openness was surrounded by certain rules, such as e.g. a tacit understanding never to correct a colleague in front of a patient. It was also stated that it was easier to handle a formal complaint from the patient than things you only had observed yourselves. Thus to dare to act could mean a confrontation with a colleague, while trying to protect a patient. Some informants said that it could also mean to dare to ask the patient about former abusive experiences in health care, and to discuss with the patient how to avoid it in future. 


\section{Figure 1.}

Core category, categories and substantive codes answering the research question; to what extent is staff aware of abuse in health care?

\section{The Core category}

The core category - 'Staff's awareness of AHC varies according to context and possibilities to act' - was derived from the interaction between five categories; Moral imagination, Relativism, Explanations, and Dissociation from AHC, and Acting against AHC. In our analyses, awareness of AHC was not a permanent state of being aware of AHC or not; all participants displayed both high and low awareness, i.e. awareness was not a feature characterizing some individuals but rather depending on the situation.

On the lower levels of awareness, Relativism was considered a higher form of awareness than the two categories Explanations, and Dissociation from AHC, because these two categories imply a resistance to acknowledge AHC. We interpreted this resistance as a struggle between awareness of AHC and a need to defend one-self. Dissociation from AHC was considered the lowest form of awareness because this category was close to disavowal.

High awareness - Moral imagination - is likely to increase the probability of Acting against AHC. However, as illustrated by an auxiliary nurse in the Moral imagination category, high awareness does not guarantee acting against AHC. I think that's horrible! she said and she told us that the patient must have felt abused when she was left uncovered in the examination chair with the gynecologist still in position for examination. She felt respect and sympathy for the patient but she did not cover the patient or ask the gynecologist to change position. The relationship between Moral imagination and Acting against AHC has to be examined further in relation to other factors that might influence staff members to act against AHC. 


\section{DISCUSSION}

Our results showed that all staff members could relate to AHC against patients.

Still, when asked, more than every second said it was difficult to come up with own, others' or general examples of AHC. When scrutinized we found that some of the given examples concerned colleagues, and only a few were own and the majority were general examples, i.e. staff is likely to be more aware of AHC involving others than themselves.

According to modern memory research there are several memory subsystems (27). While the episodic memory is detailed and colorful, close to mental reliving, semantic memory contains general information without personal experiences (28). Examples of episodic memory were rare in our interviews, and only found in the Moral imagination category, while semantic memories were common in all categories. Notable is that the informants often said that they did not know anything about AHC, and yet they talked about it and gave examples. 'Lack of memory' is a known coping strategy to suppress episodic memory and legalize semantic memory (29). It is our impression that this mechanism could have been activated in our interviews because of the taboo status of AHC. However interesting as a phenomenon, it is also a methodological weakness that heightens the risk for shallow data from the interviews. Another risk is that health care staff might feel that they ought to answer our questions in a certain e.g. ethically maintainable way, which would introduce a social desirability bias. However, our impression is that the defense mechanisms were much stronger than the social desirability in the present study. 


\section{Significance of the core category}

Staff's awareness of AHC varied from strong to weak depending on whether or not the context allowed staff to act against AHC. By context we mean structural and cultural factors such as e.g. leadership, formal/informal hierarchies, implicit and explicit rules and prevailing belief systems or implicit practices.

The fluctuation in awareness indicates that awareness might be depending on something else than the individual's resources. To take action is not always possible, e.g. in our interviews it was a tacit agreement not to criticize a colleague in front of a patient, and afterwards it might feel 'too late'. If the possibilities to act are limited, awareness can become a burden and something that is slowly shut off. On the other hand, if acting is possible and the conflict is resolved in a good way, moral imagination and acting will be positively reinforced. The notion of a possibility to act is therefore crucial for culturing moral imagination - and counteracting AHC.

It could be argued that the staff's shifting awareness of AHC could correspond to the severity of AHC. This idea was not supported by the interviews, as the informants, on the contrary, often talked about how important the 'small things' were. There were also examples of staff members, who without realizing it were taking part in severe AHC.

The implication of our core category could be interpreted in the light of what Galtung calls the vicious violence triangle $(30,31)$. According to his theory violence never occurs alone but is always legitimized by culture and/or structure, i.e. AHC is a matter of individuals, culture and structures. AHC prevention therefore has to target individual behavior and awareness as well as cultures and structures in health care. 


\section{Hindrances to act against AHC}

Our core category revealed that the characteristics of the situations in which AHC occurs might be more decisive than the degree of awareness of AHC when it comes to acting against AHC.

Even though Swedish health care is more egalitarian today, hierarchies still prevail to some extent, and might constitute a hindrance to act against AHC because it is less likely that a subordinated person speaks up for herself or for anyone else. For example, in a Norwegian study physicians were commonly seeing themselves as the experts who should decide for the patient (32). Such a notion might not be questioned by colleagues holding inferior positions. Ethicists, sociologists, and social psychologists have studied factors that contribute to conforming human beings to go with the group, even when it means to take part in actions that clearly go against generally accepted norms and their own moral appraisals (33-35). One mechanism in these theories is to create a we-against-them situation. Depersonalization can then be used to reduce 'the other's' humanity by, e.g. using a depersonalizing language, and strengthen 'our' own superiority to justify certain acts against 'them'. In health care the concept 'othering' has been used to describe the process creating staff as 'we' and patients as 'them' (36-38). A wish to maintain their privileged positions as 'we', could therefore theoretically withhold staff from acting against AHC.

Our study also showed that awareness of AHC is a complex phenomenon, and that participating in or even talking about $\mathrm{AHC}$ is a taboo. 


\section{How to counteract AHC on the basis of this study}

'Relativism', 'Explanations', and 'Dissociation from AHC' can be seen as facilitators for AHC, while the higher form of awareness of AHC found in this study, 'Moral imagination' could be used to counteract AHC. Moral imagination, however, is rarely discussed within bioethics (39).

According to Glover, moral imagination is a prerequisite for the development and maintenance of our moral recourses (33). He defines moral imagination as a way of seeing what is important for another person and argues that it can be developed. Moreover, there seems to be a general agreement that moral imagination is closely linked to the perception and ability of seeing different realities, e.g. realizing that patients can be afraid of a technical procedure that the physician uses every day $(39,40)$. These different perceptions do not have to merge, i.e. a 'double-image' can exist on a perceptual level. One of our informants also pointed out that AHC often is a matter of 'seeing' from different perspectives; 'It's simply that you have two different images of what's happening.'

How then can staff's moral imagination be developed? How can staff develop 'a reflex' to always act against AHC? An accepting attitude toward AHC, legitimizing it to be discussed, could be one way to promote development of moral imagination, i.e. develop a tolerance for different perspectives, the 'double image'.

A 'cultural change' that acknowledges AHC, and breaks the taboo is also needed. AHC has to be accepted as something that can happen in any health care encounter; i.e. health care staff need to develop a tolerance for imperfection in self and others; as suggested by Shapiro (37). This cultural change could facilitate for staff to develop confidence in handling AHC in a morally and professionally adequate way. What is possible in a first step toward a cultural change is to create an environment at the clinic, where it feels safe to admit mistakes, and give and take positive and negative critique. 


\section{CONCLUSION}

Awareness of AHC was not a feature characterizing some individuals but rather depending on the situation in which AHC occurred.

Therefore AHC prevention has to target individual behavior as well as cultures and structures in health care, e.g. there has to be a 'cultural change' that acknowledges AHC and breaks its taboo status. 


\section{REFERENCES}

1. van Campen C, Sixma H, Friele RD, Kerssens JJ, Peters L. Quality of care and patient satisfaction: a review of measuring instruments. Med Care Res Rev 1995 Mar;52(1):109-33.

2. Staniszewska SH, Henderson L. Patients' evaluations of the quality of care: influencing factors and the importance of engagement. J Adv Nurs 2005 Mar;49(5):530-7.

3. Turris SA. Unpacking the concept of patient satisfaction: a feminist analysis. J Adv Nurs 2005 May;50(3):293-8.

4. Rogers A, Karlsen S, Addington-Hall J. 'All the services were excellent. It is when the human element comes in that things go wrong': dissatisfaction with hospital care in the last year of life. J Adv Nurs 2000 Apr;31(4):768-74.

5. Hornsten A, Lundman B, Selstam EK, Sandstrom H. Patient satisfaction with diabetes care. J Adv Nurs 2005 Sep;51(6):609-17.

6. Coyle J, Williams B. Seeing the wood for the trees: defining the forgotten concept of patient dissatisfaction in the light of patient satisfaction research. Int J Health Care Qual Assur Inc Leadersh Health Serv 1999;12(6-7):i-ix.

7. Edwards C, Titchen A. Research into patients' perspectives: relevance and usefulness of phenomenological sociology. J Adv Nurs 2003 Dec;44(5):450-60.

8. Coyle J, Williams B. Valuing people as individuals: development of an instrument through a survey of person-centredness in secondary care. J Adv Nurs 2001 Nov;36(3):450-9.

9. Coyle J, Williams B. An exploration of the epistemological intricacies of using qualitative data to develop a quantitative measure of user views of health care. J Adv Nurs 2000 May;31(5):1235-43. 
10. Eriksson U, Svedlund M. Struggling for confirmation--patients' experiences of dissatisfaction with hospital care. J Clin Nurs 2007 Mar;16(3):438-46.

11. Williams B. Patient satisfaction: a valid concept? Soc Sci Med 1994 Feb;38(4):509-16.

12. Hiidenhovi H, Nojonen K, Laippala P. Measurement of outpatients' views of service quality in a Finnish university hospital. J Adv Nurs 2002 Apr;38(1):59-67.

13. Annandale E, Hunt K. Accounts of disagreements with doctors. Soc Sci Med 1998 Jan;46(1):119-29.

14. Coyle J. Understanding dissatisfied users: developing a framework for comprehending criticisms of health care work. J Adv Nurs 1999 Sep;30(3):723-31.

15. Swahnberg K, Schei B, Hilden M, Halmesmaki E, Sidenius K, Steingrimsdottir T, et al. Patients' experiences of abuse in health care: a Nordic study on prevalence and associated factors in gynecological patients. Acta Obstet Gynecol Scand 2007;86(3):349-56.

16. Swahnberg K, Thapar-Bjorkert S, Bertero C. Nullified: women's perceptions of being abused in health care. J Psychosom Obstet Gynaecol 2007 Sep;28(3):161-7.

17. Swahnberg K, Wijma B, Hearn J, Thapar-Bjorkert S, Berterö C. Mentally pinioned: Men's perceptions of being abused in health care. Int J Mens Health 2009;8(1):60-71.

18. Coyle J. Exploring the meaning of 'dissatisfaction' with health care: the importance of 'personal identity threat'. Sociol Health Illn 1999;21(1):95-123.

19. Swahnberg K, Zbikowski A, Wijma B. Ethical lapses: staff's perception of abuse in health care. J Psychosom Obstet Gynaecol 2010;31(3):123-9.

20. Patton MO. Qualitative research \& evaluation methods. 3 ed. London: Sage Publications; 2002.

21. Britten N. Qualitative Research: Qualitative interviews in medical research. BMJ 1995 July 22, 1995;311(6999):251-3. 
22. Lofland J, Lofland LH. Analysing social settings. A guide to qualitative observation and analysis. 3 ed. Belmont: Wadsworth Publishing Company; 1995.

23. Glaser B. Basics of grounded theory analysis. Emergence versus forcing. Mill Valley, CA: Sociology Press; 1992.

24. Glaser B, Strauss A. The discovery of grounded theory: Strategies for qualitative research. New York: Aldine de Gruyter; 1967/1999.

25. Streubert HJ, Carpenter DR. Qualitative Research in Nursing. Advancing the Humanistic Imperative. 2 ed. Philadelphia: Lippincott; 1995/1999.

26. Glaser B. Theoretical sensitivity. 4 ed. Mill Valley, CA: Sociology Press; 1978.

27. Wieser S, Wieser HG. Event-related brain potentials in memory: correlates of episodic, semantic and implicit memory. Clin Neurophysiol 2003 Jun;114(6):1144-52.

28. Tulving E. Episodic memory: from mind to brain. Annu Rev Psychol2002;53:1-25.

29. Bowlby J. Attachment and loss: Vol. 3. Loss: Sadness and depression. New York: Basic Books; 1980.

30. Galtung J. Violence, Peace and Peace Research. J Peace Research 1969;6:167-91.

31. Galtung J. Cultural Violence. J Peace Research 1990;27(3):291-305.

32. Falkum E, Forde R. Paternalism, patient autonomy, and moral deliberation in the physician-patient relationship. Attitudes among Norwegian physicians. Soc Sci Med 2001 Jan;52(2):239-48.

33. Glover J. Humanity. A moral history of the twentieth century. London: Pimlico; 2001.

34. Zimbardo P. The Lucifer effect. Understanding how good people turn evil. New York: Random House, Inc; 2008.

35. Bandura A. Moral disengagement in the perpetration of inhumanities. Pers Soc Psychol Rev 1999;3(3):193-209. 
36. Maccallum EJ. Othering and psychiatric nursing. J Psychiatr Ment Health Nurs 2002 Feb;9(1):87-94.

37. Shapiro J. Walking a mile in their patients' shoes: empathy and othering in medical students' education. Philos Ethics Humanit Med 2008;3:10.

38. Canales MK. Othering: toward an understanding of difference. ANS Adv Nurs Sci 2000 Jun;22(4):16-31.

39. Sherwin S. Moral perception and global visions. Bioethics 2001 Jun;15(3):175-88.

40. Hick C. The art of perception: from the life world to the medical gaze and back again. Med Health Care Philos 1999;2(2):129-40. 


\section{SUMMARY STATEMENTS}

\section{Current knowledge on this subject}

Abuse in health care is more frequently reported by female than by male patients.

Abuse in health care may cause severe suffering to both female and male patients.

Abuse in health care has so far mainly been studied from a patient perspective.

\section{What this study adds}

In the present study we found that staff's awareness of abuse in health care varied from strong to weak depending on whether or not the context allowed staff to act.

The study reveals the complicity in staffs' awareness of abuse in health care and adds knowledge from a staff perspective about the context in which abuse in health care occurs, which could be used as an empirical and theoretical base for a future intervention against abuse in health care.

Declaration of interest: The authors report no conflicts of interest. 